\title{
Early Electrocardiographic Indices for Predicting Chronic Doxorubicin-Induced Cardiotoxicity: Retrospective Case Series Study
}

Toshio Kinoshita ( $\square$ kino-00039@med.toho-u.ac.jp )

Toho University Faculty of Medicine https://orcid.org/0000-0002-8692-9923

Hitomi Yuzawa

Toho University Faculty of Medicine

Kazuhiko Natori

Toho University School of Medicine

Ryo Wada

Toho University Faculty of Medicine

Shintaro Yao

Toho University Faculty of Medicine

Kensuke Yano

Toho University Faculty of Medicine

Katsuya Akitsu

Toho University Faculty of Medicine

Hideki Koike

Toho University Faculty of Medicine

Masaya Shinohara

Toho University Faculty of Medicine

Tadashi Fujino

Toho University Faculty of Medicine

Hideaki Shimada

Toho University Graduate School of Medicine

Takanori Ikeda

Toho University Faculty of Medicine

\section{Research}

Keywords: Electrocardiogram, T-wave, QTa prolongation, early detection, doxorubicin, CTRCD

Posted Date: August 6th, 2020

DOl: https://doi.org/10.21203/rs.3.rs-52751/v1 
License: (c) (i) This work is licensed under a Creative Commons Attribution 4.0 International License. Read Full License 


\section{Abstract}

Background: Dealing with chemotherapy-related cardiac dysfunction (CTRCD) remains a significant problem complicated by the difficulty in early detection of cardiotoxicity. Electrocardiogram (ECG) is expected to be the most realistic methodology due to lower cost-performance and non-invasiveness. We investigated the long-term visual fluctuations in the ECG waveforms in patients with chronic doxorubicin (DOX)-induced cardiotoxicity to identify ECG indices for the early detection of cardiotoxicity.

Methods: We conducted a retrospective case series study by reviewing the medical records of 470 consecutive patients with malignant lymphoma who were treated with DOX at our institute between January 2010 and December 2017. Of them, 23 (4.9\%) patients developed left ventricular dysfunction and were diagnosed with CTRCD using echocardiography. We assessed the ECG indices on 12-lead ECG recordings before and after treatment in 15 patients; eight patients were excluded due to conduction disturbances or atrial fibrillation.

Results: CTRCD was detected at a median of 475 (interquartile range, IQR: $341-1333$ ) days after initiating chemotherapy. The evaluation of ECG indices preceding CTRCD development was performed 93 (IQR: 52-232) days before the detection of CTRCD. In the stage of CTRCD, the most significant ECG change was T-wave flattening in leads V3-V6 (12 patients, 80\%). Additionally, QTa prolongation was observed in leads I and aVL ( $n=10,66 \%)$, leads II, III, and aVF ( $\mathrm{n}=9,60 \%)$, and leads V3-V6 $(n=10,73 \%)$. These ECG changes were not observed before the treatment but were detected mildly in the pre-CTRCD stage, which subsequently worsened in the CTRCD stage.

Conclusions: This study indicated that T-wave changes and QTa prolongation may be useful as an early indicator before the onset of CTRCD in patients with DOX-induced cardiotoxicity.

\section{Introduction}

Anthracyclines are among the most diffused chemotherapeutic agents, and they still represent the base of treatment in many patients with cancer $[1,2]$. Anthracyclines can result in acute, chronic, and late cardiotoxicity; they represent the greatest risk [3] for cancer therapeutics-related cardiac dysfunction (CTRCD) $[4,5]$. Currently, early- and late-onset chronic cardiotoxicities are considered irreversible and result in poor prognoses, which remains a significant problem and is complicated by the difficulty in the early detection of the onset of CTRCD. Some of the following reasons account for this difficulty: CTRCD can occur for an extended period after treatment; examination cost-performance and non-invasiveness; and follow-up observations by non-cardiologists. Electrocardiogram (ECG) is expected to be the most realistic methodology to overcome these problems. Numerous investigators have evaluated the relevance between ECG indices, almost QT prolongation, and life-threatening arrhythmia [6, 7]; however, almost none of the studies have focused on the usefulness of ECG indices in left ventricular dysfunction.

Doxorubicin (DOX), one of the representative anthracyclines, has been used as a chemotherapeutic agent in patients with malignant lymphoma. In this study, we investigated the long-term visual fluctuations in 
the ECG waveform in patients with chronic DOX-induced cardiotoxicity and sought to identify ECG indices for the early detection of such cardiotoxicity.

\section{Methods}

\section{Study population}

This retrospective single-center cohort study included 470 consecutive patients with malignant lymphoma who were admitted to the Toho University Omori Medical Center, Tokyo, Japan, between January 2010 and December 2017. These patients were treated with DOX. Of them, 23 (4.8\%) patients developed left ventricular dysfunction that meets the criteria for CTRCD as described below without a history of ischemic heart disease (IHD), idiopathic cardiomyopathy, including dilated cardiomyopathy, hypertrophic cardiomyopathy, valvular heart disease, and myocarditis. These patients were diagnosed with CTRCD. Finally, we assessed 15 patients using sequential 12-leads ECG recordings, except eight patients who had atrial tachyarrhythmias, including atrial fibrillation, bundle branch block, and permanent pacing, which result in unevaluable ECG indices. We examined the clinical indices, treatment histories, and echocardiographic data, and analyzed the ECG waveforms in these patients.

This study was approved by the Ethics Committee of Toho University Omori Medical Center (approval number: M19111). Informed consent was obtained in the form of an opt-out option on the website of Toho University Omori Medical Center. The study was performed in accordance with the Declaration of Helsinki.

\section{Echocardiography and visual ECG analysis}

Left ventricular end-diastolic diameter (LVDd) and ejection fraction (LVEF) were estimated with twodimensional guided M-mode echocardiography [8] using the ultrasound system (GE Vivid $i$; GE Healthcare Japan, Tokyo, Japan). The peak velocities of early diastolic filling wave (E-wave) and atrial contraction wave ( $A$-wave) in addition to the isovolumic relaxation time of the left ventricle were measured with the records of transmitral flow velocity patterns. The $E / A$ ratios were calculated with the peak velocities of $E$ wave and A-wave. CTRCD criteria defined by the American Society of Echocardiography and European Association of Cardiovascular Imaging [9] include LVEF > 53\% before cancer therapy and decrease by > $10 \%$ after chemotherapy.

ECG waveforms were analyzed using a resting 12-lead surface ECG (Cardiofax, NIHON KOHDEN, Tokyo, Japan). The ECGs were recorded at a paper speed of $25 \mathrm{~mm} / \mathrm{s}$ and $10 \mathrm{mV} / \mathrm{mm}$ standardization. The ECG indices were assessed by two cardiologists blinded to the clinical status of the patient. We divided the 12lead into the following sets: V1 and V2, V3-V6, I and aVL, and II, III, and aVF. Subsequently, we visually evaluated the fluctuations in the waveforms of the following indices: amplitude of QRS complex and Twave, prolongation of PQ, QRS duration, QT interval, QTa interval, and ST. Visually definite changes in ECG indices were admitted in significant fluctuations. QTa, the QT apex interval, was defined as the interval between the onset of the QRS complex and the apex of the T-wave. The pre-CTRCD stage was 
defined as the stage with normal LVEF immediately before CTRCD was detected. The days between the pre-CTRCD and CTRCD stages were calculated.

\section{Statistical analysis}

Data of the age, LVEF, and LVDd are expressed as mean \pm standard deviation. Data regarding serum brain natriuretic peptide (BNP) levels and time durations are presented as median with first and third quartiles (25\%-75\%). Echocardiographic data among the periods of pre-treatment, pre-CTRCD, and CTRCD were compared using one-way repeated measures analysis of variance (ANOVA). Statistical analyses were performed using EZR (Saitama Medical Center, Jichi Medical University, Saitama, Japan), which is a graphical user interface for R (The R Foundation for Statistical Computing, Vienna, Austria). EZR is a modified version of $\mathrm{R}$ commander designed to add statistical functions frequently used in biostatistics [10]. $P$-values $<0.05$ were considered statistically significant.

\section{Results}

\section{Patients characteristics}

The clinical characteristics of the 15 patients with CTRCD are summarized in Table 1. Their mean age was $57 \pm 12$ years, and 10 (66\%) patients were male. Five (33\%), $3(20 \%), 4(26 \%)$, and $3(20 \%)$ patients suffered from hypertension, dyslipidemia, diabetes mellitus, and chronic kidney disease, respectively. LVEF and serum BNP levels before the treatment were normal. Table 2 summarizes the details of the treatments; 11 (73\%) patients were treated with the R-CHOP regimen, and all patients received DOX. The mean total dose of each anticancer drug was as follows: DOX, $317 \pm 65 \mathrm{mg} / \mathrm{m}^{2}$; cyclophosphamide, $4,696 \pm 909 \mathrm{mg} / \mathrm{m}^{2}$; vincristine, $8.1 \pm 1.7 \mathrm{mg} / \mathrm{m}^{2}$; and rituximab, $2,565 \pm 468 \mathrm{mg} / \mathrm{m}^{2}$. One (6.7\%) patient underwent radiation therapy including the heart.

\section{Time to detection of CTRCD and echocardiographic evaluation}

Table 3 summarizes the duration between the initiation of chemotherapy and the detection of CTRCD. Within 6 months (68 [IQR, 65-116] days), CTRCD was observed in $3(20 \%)$ patients. After the completion of 6 or 8 courses of the CHOP or R-CHOP regimen, 3 (20\%) patients developed CTRCD within 6-12 months (346 [IQR, 269-354] days). Between 12 and 36 months (453 [IQR, 412-497] days), 3 (20\%) patients developed CTRCD. After 36 months (1332 [IQR, 1176-1983] days), CTRCD was detected in 6 (40\%) patients. The results of the echocardiographic evaluations are summarized in Table 4.

Echocardiography and ECG at the time of detection of CTRCD were performed at a median of 475 (IQR, 341-1333) days after the initiation of chemotherapy. Furthermore, the evaluation preceding the detection of CTRCD (pre-CTRCD stage) was performed 93 (IQR, 52-232) days before. Figure 1 illustrates the fluctuation in heart rate and deterioration of left ventricular function at each stage. Heart rate had significantly decreased in the pre-CTRCD stage compared with that in the pre-treatment stage (84.7 \pm 12 vs. $98.7 \pm 12 \mathrm{bpm}, P<0.05)$. LVEF at the time of detection of CTRCD was significantly lower than that in 
the pre-CTRCD stage and baseline ( $43.6 \%$ vs. $62.1 \%$ and $66.8 \%$, respectively, $P<0.05)$. Left ventricular diastolic function represented by $E / A$ revealed no significant difference among the stages.

\section{Visual ECG evaluation of CTRCD}

Table 5 and Fig. 2 demonstrate the visual ECG changes between the pre-CTRCD and CTRCD stages. In the CTRCD stage, the primary ECG changes included T-wave flattening in leads V3-V6 $(n=12 / 15$ patients, 80\%). QTa prolongation was recognized in leads I and aVL, II, III, and aVF, and V3-V6 (10 [66\%], 9 [60\%], and 11 [73\%] patients, respectively). Interestingly, increases in QRS amplitude in leads V3-V6 were also observed in 7 (47\%) patients. In contrast, decreases in QRS amplitude were only approximately $20 \%$ in all leads. T-wave flattening in leads I and aVL, II, III, and aVF, and V1 and V2 were observed in 8 (53\%), 5 (33\%), and 7 (47\%) patients, respectively. Interestingly, T-wave flattening in leads V3-V6 had already been observed in the pre-CTRCD stage in $5(45 \%)$ patients. Furthermore, in the pre-CTRCD stage, QTa prolongation had already been observed in approximately $40 \%$ of all leads. Increases in QRS amplitude in leads V3-V6 also had been observed in 6 (55\%) patients. Figure 3 illustrates the representative visual ECG fluctuations in patients with CTRCD.

\section{Discussion}

The present study demonstrated the time course of ECG fluctuations in human chronic DOX-induced cardiotoxicity. T-wave changes, including flattening and inversion, and QTa prolongation were the commonest findings in our study population. These ECG fluctuations had been detected mildly in the preCTRCD stage, which subsequently worsened in the CTRCD stage.

\section{Heart rate, $\mathrm{P}$ wave, and $\mathrm{PQ}$ duration change}

In our study, heart rate was significantly decreased in the pre-CTRCD and CTRCD stages compared with the pre-treatment stage. Several studies reported that DOX appears to demonstrate a negative chronotropic effect [11-13]. Although the exact mechanism remains unclear, oxidized calcium/calmodulin-dependent protein kinase II has been reported to be associated with sinoatrial node fibrosis and apoptosis [14]. Regarding $P$ wave changes, ectopic atrial rhythms were found in a few cases in the CTRCD stage, which is consistent with negative chronotropic effects. Some reports have demonstrated the suppression of AV conduction in DOX treatment and reported that type I atrioventricular block was more frequently detected in those with higher cumulative doses of DOX $[15,16]$. However, in our study, PQ prolongation was found in only $1 / 15$ cases.

\section{QRS complex duration and amplitude change}

In our study, there were no cases of the prolonged QRS complex. Several studies reported on the widening of the QRS complex following DOX exposure, which suggested intraventricular conduction defects due to myocardial ultrastructural changes, such as intercalated disc separation, myofibrillar derangement, vacuolization, and interstitial edema $[11,17]$. Furthermore, Pecoraro et al. demonstrated that DOX 
treatment resulted in markedly reduced expression and function of connexin-43, which potentially leads to slower conduction velocity and loss of rapid electrical synchronization throughout the myocardium [18]. However, there are inconsistent findings in the literature. Ducroq et al. demonstrated no remarkable changes in the duration of the QRS complex in acute perfused isolated guinea pig hearts with DOX treatment [12]. Considering that a majority of the data on QRS duration was derived from animal studies, these discrepancies may suggest that the depolarization abnormalities are relatively mild in humans or that the mechanism of DOX-induced cardiotoxicity in the chronic phase in humans may be different from that in animals, especially, rats. Regarding the amplitude of the QRS complex, previous reports have demonstrated a decrease in the amplitude of the QRS complex $[19,20]$. However, in our study, an increase in the amplitude was observed in leads V3-V6, and a decrease was observed in leads V1-V2 in both CTRCD and pre-CTRCD stages. Several studies, including animal and human patient studies, demonstrated that the left ventricle developed compensatory hypertrophy in anthracycline cardiomyopathy [21-23]. In our study, although echocardiographic results revealed no significant hypertrophic changes in both pre-CTRCD and CTRCD stages, ECG results may indicate the early stage of compensatory hypertrophy.

\section{QTa prolongation and T-wave flattening}

Drug-induced QT prolongation is an established research area, and numerous studies have been conducted to elucidate the mechanisms and pathogenesis of the QT interval concerning anticancer drugs, especially anthracyclines $[6,7,24]$. However, the clinical consequences of QT prolongation, such as arrhythmias or sudden cardiac death, remain rare [24]. Furthermore, Nousiainen et al. demonstrated that $18 \%$ of patients exposed to DOX experienced QTc prolongation of greater than $50 \mathrm{~ms}$ and that these changes were independent of LV function [25]. Therefore, the utility of only QTC as a predictor of arrhythmia risk assessment and cardiac dysfunction may be limited. Visual evaluation of QT prolongation is difficult to determine the end of the T-wave and to make corrections for the heart rate. Therefore, in terms of measurement technology, visual evaluation of the QT interval is indefinite. Whereas several reports have revealed that QTa prolongation occurring with DOX treatment is due to the lengthening of the action potentials of ventricular cardiomyocytes $[11,26]$ and is correlated with the severity of the histological lesions of the heart [27]. Practically, in our study, mild QTa prolongation had already been observed in some patients in the pre-CTRCD stage and several patients in the CTRCD stage. Therefore, QTa prolongation may be an extremely important finding in the early detection of DOX-induced myocardial damage. T-wave flattening in the CTRCD stage was widely observed in many leads, especially V3-V6. This trend was also observed in the pre-CTRCD stage, albeit weakly. To date, T-wave changes due to myocardial damage caused by anticancer drugs have not been reported in incoherent human clinical cases. Although the data are not on anticancer drugs, Tuohinen et al. reported T-wave changes induced by radiotherapy in patients with breast cancer [28]. The mean heart dose of radiotherapy was independently associated with both the decline and inversion of the T-wave. They hypothesized that the primary mechanisms include fibrotic tissue changes induced by epicardial inflammatory effects and myocardial edema. ECG alterations are the most reliable and consistent in DOX-treated rodents [11, 26, 29]. Notably, the development of DOX cardiomyopathy in rats is accompanied by T-wave flattening [11, 
$17,26]$, and progressive QTa prolongation $[11,27,30]$. These findings are consistent with our results. In a rat model, Kharin et al. have described a highly essential report on ventricular repolarization heterogeneities in the chronic phase of DOX cardiomyopathy with a focus on the ventricular activation recovery interval (ARI) [31, 32]. The major findings of these studies suggested that (1) ARIs of the epicardium of both the right and left ventricles were significantly prolonged and (2) the inhomogeneous prolongation of ARIs resulted in (i) increases in the epicardial right ventricular and overall ARI dispersions and (ii) inhomogeneous alterations of the regional ARI gradients across the ventricular epicardium.

\section{Timing of ECG fluctuations in CTRCD progression}

In our study, the median time of detection of CTRCD was 453 (IQR, 269-1212) days, and 6/15 patients developed it after at least 3 years. Therefore, our study population generally represented early or lateonset chronic cardiotoxicity. Pre-CTRCD status was identified approximately 93 (IQR, 52-232) days before the CTRCD status, and despite the absence of LV dysfunction, QTa prolongation and T-wave flattening could be recognized 2-3 months before the onset of CTRCD. Till now, there are no studies that have investigated the ECG fluctuations in detail in patients with anthracycline-derived CTRCD. Our results demonstrated that CTRCD develops within a few months of the appearance of QTa prolongation and Twave flattening. Therefore, we suggest that the follow-up ECG should be performed approximately at least once every 3-6 months in patients treated with DOX.

Considering the evidence that patients who begin cardioprotective therapy more than 6 months after the onset of anthracycline-induced cardiomyopathy would be a non-responder, LVEF recovery depends on the early detection of cardiotoxicity and prompt heart failure treatment [33]. To facilitate an approach for the prevention, detection, and management of CTRCD, Abdel et al. suggested that it is useful to think of CTRCD along the spectrum of the American College of Cardiology (ACC)/American Heart Association (AHA) stages of heart failure [34]. In our study, the pre-CTRCD stage corresponds to AHA stage A or B1, and it is extremely essential to identify high-risk patients in these stages.

\section{Study Limitations}

In the present study, we only focused on the evaluation of the visual appearance of the standard 12-lead ECG. A more detailed evaluation of the depolarization and repolarization indices at the micro-potential level, such as ventricular late potentials and T-wave alternans, are not available. In terms of early detection of myocardial damage, we did not compare with other modalities, such as serum troponin I, global longitudinal strain, and multigated acquisition scans. Therefore, prospective comparisons of the abilities of these modalities in the early detection of CTRCD are needed in the future.

\section{Conclusions}

The present study demonstrated the temporal ECG fluctuations in chronic DOX-induced cardiotoxicity. Twave changes and QTa prolongation were the most important findings in our study population and may help in the early detection of the onset of CTRCD. In the future, large prospective studies are needed to 
revalidate the results of this study and compare the abilities of multiple modalities in the early detection of CTRCD.

\section{Abbreviations}

BMI: body mass index; CTRCD: chemotherapy-related cardiac dysfunction; DOX: Doxorubicin; ECG: electrocardiogram, electrocardiographic; IHD: ischemic heart disease; IQR: interquartile range; LVDd: left ventricular end-diastolic diameter; LVEF = left ventricular ejection fraction

\section{Declarations}

\section{Acknowledgments}

We would like to thank the staff of the Division of Hematology and Oncology and the Department of Internal Medicine at the Toho University Omori Medical Center for their assistance with the study. We also appreciate the staff of the Cancer Center of the Toho University Omori Medical Center for providing the data.

\section{Authors' contributions}

TK and HY have led the study from its inception to final revisions. RW and KA made substantial contribution to data acquisition and analysis. HK and MS led statistical analysis. SY, KY and TF contributed to acquisition of data, analysis of data. TI contributed to study conception and design, drafting of manuscript, and editing of manuscript. HS and KN contributed to acquisition of data, drafting of manuscript, and editing of manuscript. All authors read and approved the final manuscript.

\section{Funding}

The authors declare that there is no funding for any portion of this research.

\section{Availability of data and materials}

The datasets used and analyzed during the current study are available from the corresponding author on reasonable request.

\section{Ethics approval and consent to participate}

This study was approved by the Ethics Committee of Toho University Omori Medical Center (approval number: M19111). Informed consent was obtained in the form of an opt-out option on the website of Toho University Omori Medical Center. The study was performed in accordance with the Declaration of Helsinki.

\section{Consent for publication}


Not applicable.

\section{Competing interests}

All authors declare that there are no potential conflicts of interest.

\section{Author details}

${ }^{1}$ Department of Cardiovascular Medicine, Toho University Faculty of Medicine, 6-11-1 Omori-Nishi, Ohta$\mathrm{Ku}$, Tokyo, Japan. ${ }^{2}$ Division of Hematology and Oncology, Department of Internal Medicine, Toho University School of Medicine, 6-11-1 Omori-Nishi, Ohta-Ku, Tokyo, Japan. ${ }^{3}$ Department of Gastroenterological Surgery and Clinical Oncology, Graduate School of Medicine, Toho University, 6-11-1 Omori-Nishi, Ohta-Ku, Tokyo, Japan.

\section{References}

1. Octavia Y, Tocchetti CG, Gabrielson KL, Janssens S, Crijns HJ, Moens AL. Doxorubicin-induced cardiomyopathy: from molecular mechanisms to therapeutic strategies. J Mol Cell Cardiol. 2012;52(6):1213-25.

2. Henriksen PA. Anthracycline cardiotoxicity: an update on mechanisms, monitoring and prevention. Heart. 2018;104(12):971-7.

3. Jones RL, Swanton C, Ewer MS. Anthracycline cardiotoxicity. Expert Opin Drug Saf. 2006;5(6):791809.

4. Cardinale D, lacopo F, Cipolla CM. Cardiotoxicity of Anthracyclines. Front Cardiovasc Med. 2020;7:26.

5. Saleh Y, Abdelkarim O, Herzallah K, Abela GS. Anthracycline-induced cardiotoxicity: mechanisms of action, incidence, risk factors, prevention, and treatment. Heart Fail Rev. 2020. doi:10.1007/s10741020-09968-2.

6. Roden DM. A current understanding of drug-induced QT prolongation and its implications for anticancer therapy. Cardiovasc Res. 2019;115(5):895-903.

7. Duan J, Tao J, Zhai M, Li C, Zhou N, Lv J, et al. Anticancer drugs-related QTc prolongation, torsade de pointes and sudden death: current evidence and future research perspectives. Oncotarget. 2018;9(39):25738-49.

8. Teichholz LE, Kreulen T, Herman MV, Gorlin R. Problems in echocardiographic volume determinations: echocardiographic-angiographic correlations in the presence of absence of asynergy. Am J Cardiol. 1976;37(1):7-11.

9. Plana JC, Galderisi M, Barac A, Ewer MS, Ky B, Scherrer-Crosbie M, et al. Expert consensus for multimodality imaging evaluation of adult patients during and after cancer therapy: a report from the American Society of Echocardiography and the European Association of Cardiovascular Imaging. Eur Heart J Cardiovasc Imaging. 2014;15(10):1063-93. 
10. Kanda Y. Investigation of the freely available easy-to-use software 'EZR' for medical statistics. Bone Marrow Transplant. 2013;48(3):452-8.

11. Jensen RA, Acton EM, Peters JH. Doxorubicin cardiotoxicity in the rat: comparison of electrocardiogram, transmembrane potential, and structural effects. J Cardiovasc Pharmacol. 1984;6(1):186-200.

12. Ducroq J, Moha ou Maati H, Guilbot S, Dilly S, Laemmel E, Pons-Himbert C, et al. Dexrazoxane protects the heart from acute doxorubicin-induced QT prolongation: a key role for I(Ks). $\mathrm{Br} \mathrm{J}$ Pharmacol. 2010;159(1):93-101.

13. Xin Y, Zhang S, Gu L, Liu S, Gao H, You Z, et al. Electrocardiographic and biochemical evidence for the cardioprotective effect of antioxidants in acute doxorubicin-induced cardiotoxicity in the beagle dogs. Biol Pharm Bull. 2011;34(10):1523-6.

14. Wu Y, Anderson ME. CaMKII in sinoatrial node physiology and dysfunction. Front Pharmacol. 2014;5:48.

15. Larsen RL, Jakacki RI, Vetter VL, Meadows AT, Silber JH, Barber G. Electrocardiographic changes and arrhythmias after cancer therapy in children and young adults. Am J Cardiol. 1992;70(1):73-7.

16. Kilickap S, Akgul E, Aksoy S, Aytemir K, Barista I. Doxorubicin-induced second degree and complete atrioventricular block. Europace. 2005;7(3):227-30.

17. Agen C, Bernardini N, Danesi R, Della Torre P, Costa M, Del Tacca M. Reducing doxorubicin cardiotoxicity in the rat using deferred treatment with ADR-529. Cancer Chemother Pharmacol. 1992;30(2):95-9.

18. Pecoraro M, Rodriguez-Sinovas A, Marzocco S, Ciccarelli M, laccarino G, Pinto A, et al. Cardiotoxic Effects of Short-Term Doxorubicin Administration: Involvement of Connexin 43 in Calcium Impairment. Int J Mol Sci. 2017; 18(10):10.3390/ijms18102121.

19. Horacek JM, Jakl M, Horackova J, Pudil R, Jebavy L, Maly J. Assessment of anthracycline-induced cardiotoxicity with electrocardiography. Exp Oncol. 2009;31(2):115-7.

20. Roberts WC, Haque S, Hall SA. Total 12-Lead QRS Voltage in Patients Having Orthotopic Heart Transplantation for Heart Failure Caused by Adriamycin-Induced Cardiomyopathy. Cardiology. 2018;141(3):172-5.

21. Rodrigues PG, Miranda-Silva D, Costa SM, Barros C, Hamdani N, Moura C, et al. Early myocardial changes induced by doxorubicin in the nonfailing dilated ventricle. Am J Physiol Heart Circ Physiol. 2019;316(3):H459-75.

22. Du Q, Zhu B, Zhai Q, Yu B. Sirt3 attenuates doxorubicin-induced cardiac hypertrophy and mitochondrial dysfunction via suppression of Bnip3. Am J Transl Res. 2017;9(7):3360-73.

23. Katamadze NA, Lartsuliani KP, Kiknadze MP. Left ventricular function in patients with toxic cardiomyopathy and with idiopathic dilated cardiomyopathy treated with Doxorubicin. Georgian Med News. 2009; (166):43-48.

24. Porta-Sanchez A, Gilbert C, Spears D, Amir E, Chan J, Nanthakumar K, et al. Incidence, Diagnosis, and Management of QT Prolongation Induced by Cancer Therapies: A Systematic Review. J Am Heart 
Assoc. 2017; 6(12):10.1161/JAHA.117.007724.

25. Nousiainen T, Vanninen E, Rantala A, Jantunen E, Hartikainen J. QT dispersion and late potentials during doxorubicin therapy for non-Hodgkin's Iymphoma. J Intern Med. 1999;245(4):359-64.

26. Jensen RA, Acton EM, Peters JH. Electrocardiographic and transmembrane potential effects of 5iminodaunorubicin in the rat. Cancer Res. 1984;44(9):4030-9.

27. Cirillo R, Sacco G, Venturella S, Brightwell J, Giachetti A, Manzini S. Comparison of doxorubicin- and MEN 10755-induced long-term progressive cardiotoxicity in the rat. J Cardiovasc Pharmacol. 2000;35(1):100-8.

28. Tuohinen SS, Keski-Pukkila K, Skyttä T, Huhtala H, Virtanen V, Kellokumpu-Lehtinen P, et al. Radiotherapy-induced Early ECG Changes and Their Comparison with Echocardiography in Patients with Early-stage Breast Cancer. Anticancer Res. 2018;38(4):2207-15.

29. van Acker SA, Kramer K, Voest EE, Grimbergen JA, Zhang J, van der Vijgh, et al. Doxorubicin-induced cardiotoxicity monitored by ECG in freely moving mice. A new model to test potential protectors. Cancer Chemother Pharmacol. 1996;38(1):95-101.

30. Xu M, Sheng L, Zhu X, Zeng S, Chi D, Zhang GJ. Protective effect of tetrandrine on doxorubicininduced cardiotoxicity in rats. Tumori. 2010;96(3):460-4.

31. Kharin SN, Krandycheva VV, Strelkova MV, Tsvetkova AS, Shmakov DN. Doxorubicin-induced changes of ventricular repolarization heterogeneity: results of a chronic rat study. Cardiovasc Toxicol. 2012;12(4):312-7.

32. Kharin S, Krandycheva V, Tsvetkova A, Strelkova M, Shmakov D. Remodeling of ventricular repolarization in a chronic doxorubicin cardiotoxicity rat model. Fundam Clin Pharmacol. 2013;27(4):364-72.

33. Cardinale D, Colombo A, Lamantia G, Colombo N, Civelli M, De Giacomi G, et al. Anthracyclineinduced cardiomyopathy: clinical relevance and response to pharmacologic therapy. J Am Coll Cardiol. 2010;55(3):213-20.

34. Abdel-Qadir H, Amir E, Thavendiranathan P. Prevention, Detection, and Management of Chemotherapy-Related Cardiac Dysfunction. Can J Cardiol. 2016;32(7):891-9.

\section{Tables}

Table 1. Patient characteristics CTRCD, cancer therapeutics-related cardiac dysfunction; BMI, body mass index; CCr, creatinine clearance; HbA1c, hemoglobin A1c; BNP, brain natriuretic peptide; LVEF, left ventricular ejection fraction; IQR, interquartile range 


\begin{tabular}{|ll|}
\hline CTRCD cases & Overall $\mathrm{n}=15$ \\
\hline Clinical indices & \\
\hline Age, yrs & $57 \pm 12$ \\
\hline Male, $\mathrm{n}(\%)$ & $10(66)$ \\
\hline BMl & $22.2 \pm 4.2$ \\
\hline Hypertension, $\mathrm{n}(\%)$ & $5(33)$ \\
\hline Dyslipidemia, $\mathrm{n}(\%)$ & $3(20)$ \\
\hline Diabetes mellitus, $\mathrm{n}(\%)$ & $4(26)$ \\
\hline Chronic kidney disease, $\mathrm{n}(\%)$ & $3(20)$ \\
\hline Serum biomarkers & \\
\hline CCr & $99.4 \pm 53$ \\
\hline Hemoglobin (g/dl) & $11.9 \pm 2.6$ \\
\hline HbA1c (\%) & $5.7 \pm 0.8$ \\
\hline BNP (pg/ml) & $18(\mathrm{IQR} 12-42)$ \\
\hline LVEF (\%) & $66.8 \pm 6.4$ \\
\hline
\end{tabular}

Table 2. Treatment history and total dose of anticancer agents

R-CHOP regimen includes rituximab, cyclophosphamide, doxorubicin, vincristine, and prednisolone. ABVD regimen includes doxorubicin, bleomycin, vinblastine, and dacarbazine. 


\begin{tabular}{|ll|}
\hline Treatment & Overall $\mathrm{n}=15$ \\
\hline Regimen & \\
\hline R-CHOP, $\mathrm{n}(\%)$ & $11(74)$ \\
\hline CHOP, $\mathrm{n}(\%)$ & $2(13)$ \\
\hline ABVD, $\mathrm{n}(\%)$ & $2(13)$ \\
\hline Anticancer agents & \\
\hline Doxorubicin, $\mathrm{n}(\%)$ & $15(100)$ \\
\hline Cyclophosphamide, $\mathrm{n}(\%)$ & $13(87)$ \\
\hline Vincristine, $\mathrm{n}(\%)$ & $13(87)$ \\
\hline Rituximab, $\mathrm{n}(\%)$ & $11(73)$ \\
\hline Cumulative dose of anticancer agents & \\
\hline Doxorubicin $\left(\mathrm{mg} / \mathrm{m}^{2}\right)$ & $317 \pm 65$ \\
\hline Cyclophosphamide $\left(\mathrm{mg} / \mathrm{m}^{2}\right)$ & $4696 \pm 909$ \\
\hline Vincristine $\left(\mathrm{mg} / \mathrm{m}^{2}\right)$ & $8.1 \pm 1.7$ \\
\hline Rituximab $\left(\mathrm{mg} / \mathrm{m}^{2}\right)$ & $2565 \pm 468$ \\
\hline Radiation therapy including the heart, $\mathrm{n}(\%)$ & $1(7)$ \\
\hline & \\
\hline
\end{tabular}

Table 3. Time till the detection of CTRCD

CTRCD, cancer therapeutics-related cardiac dysfunction; M, month; IQR, interquartile range

\begin{tabular}{|lll|}
\hline Period (month) & $\mathrm{n}=15$ & Days \\
\hline Overall, n (\%) & $15(100)$ & 453 (IQR 269-1212) \\
\hline$-6 \mathrm{M}, \mathrm{n}(\%)$ & $3(20)$ & 68 (IQR 65-116) \\
\hline $6-12 \mathrm{M}, \mathrm{n}(\%)$ & $3(20)$ & 346 (IQR 269-354) \\
\hline $12-36 \mathrm{M}, \mathrm{n}(\%)$ & $3(20)$ & 453 (IQR 412-497) \\
\hline $36-\mathrm{M} \mathrm{n}(\%)$ & $6(40)$ & 1332 (IQR 1176-1983) \\
\hline
\end{tabular}


Table 4. Echocardiographic evaluations and duration since the initiation of chemotherapy

LVEF, left ventricular ejection fraction; LVDd, left ventricular end-diastolic diameter; IQR, interquartile range

\begin{tabular}{|llll|}
\hline & Baseline & pre-CTRCD stage & CTRCD stage \\
\hline Period from commencement & $\square$ & 319 (IQR 179-992) & 475 (IQR 341-1333) \\
\hline of chemotherapy (days) & & & \\
\hline LVEF (\%) & $66.8 \pm 6.4$ & $62.1 \pm 6.8$ & $43.6 \pm 9.6$ \\
\hline LVDd (mm) & $46.6 \pm 6.8$ & $49.2 \pm 5.9$ & $51.4 \pm 7.3$ \\
\hline E/A ratio & $0.92 \pm 0.33$ & $0.81 \pm 0.22$ & $1.11 \pm 0.90$ \\
\hline
\end{tabular}

Table 5. ECG changes in the pre-CTRCD and CTRCD stages

ECG, electrocardiogram; CTRCD, cancer therapeutics-related cardiac dysfunction 


\begin{tabular}{|c|c|c|c|c|c|c|c|c|}
\hline & & \multicolumn{4}{|c|}{ pre-CTRCD stage, $n=11$} & \multicolumn{3}{|c|}{$\begin{array}{l}\text { CTRCD stage, } \\
\mathrm{n}=15\end{array}$} \\
\hline ECG indices & I.aVL & II.III.aVF & V1.V2 & $\begin{array}{l}\text { V3- } \\
\text { V6 }\end{array}$ & I.aVL & II.III.aVF & V1.V2 & $\begin{array}{l}\text { V3- } \\
\text { V6 }\end{array}$ \\
\hline $\mathrm{P}$ wave change, $\mathrm{n}(\%)$ & $1(9)$ & $1(9)$ & 0 & $\begin{array}{l}1 \\
(10)\end{array}$ & 0 & $3(20)$ & 0 & $\begin{array}{l}2 \\
(13)\end{array}$ \\
\hline PQ prolongation, n (\%) & $1(9)$ & $1(9)$ & 0 & $\begin{array}{l}1 \\
(10)\end{array}$ & 0 & $1(7)$ & 0 & $\begin{array}{l}1 \\
(7)\end{array}$ \\
\hline $\begin{array}{l}\text { QRS duration prolongation, } \\
\mathrm{n}(\%)\end{array}$ & 0 & 0 & 0 & 0 & 0 & 0 & 0 & 0 \\
\hline $\begin{array}{l}\text { QRS amplitude increase, } \mathrm{n} \\
(\%)\end{array}$ & $\begin{array}{l}3 \\
(27)\end{array}$ & $2(18)$ & $2(18)$ & $\begin{array}{l}6 \\
(55)\end{array}$ & $\begin{array}{l}3 \\
(20)\end{array}$ & $1(7)$ & $3(20)$ & $\begin{array}{l}7 \\
(47)\end{array}$ \\
\hline $\begin{array}{l}\text { QRS amplitude decrease, } n \\
(\%)\end{array}$ & $1(9)$ & $2(18)$ & $4(36)$ & $\begin{array}{l}1 \\
(9)\end{array}$ & $\begin{array}{l}3 \\
(20)\end{array}$ & $4(27)$ & $4(27)$ & $\begin{array}{l}3 \\
(20)\end{array}$ \\
\hline QTa prolongation, n (\%) & $\begin{array}{l}5 \\
(45)\end{array}$ & $5(45)$ & $4(36)$ & $\begin{array}{l}5 \\
(45)\end{array}$ & $\begin{array}{l}10 \\
(66)\end{array}$ & $9(60)$ & $8(53)$ & $\begin{array}{l}11 \\
(73)\end{array}$ \\
\hline QT prolongation, n (\%) & 0 & $3(27)$ & 0 & $\begin{array}{l}2 \\
(20)\end{array}$ & $\begin{array}{l}2 \\
(13)\end{array}$ & $3(20)$ & $1(7)$ & $\begin{array}{l}4 \\
(27)\end{array}$ \\
\hline T wave flattening, $\mathrm{n}(\%)$ & $\begin{array}{l}2 \\
(18)\end{array}$ & $3(27)$ & $3(27)$ & $\begin{array}{l}5 \\
(45)\end{array}$ & $\begin{array}{l}8 \\
(53)\end{array}$ & $5(33)$ & $7(47)$ & $\begin{array}{l}12 \\
(80)\end{array}$ \\
\hline T wave inversion, $\mathrm{n}(\%)$ & 0 & $1(9)$ & $1(9)$ & $\begin{array}{l}2 \\
(20)\end{array}$ & $\begin{array}{l}2 \\
(13)\end{array}$ & $2(13)$ & $4(27)$ & $\begin{array}{l}6 \\
(40)\end{array}$ \\
\hline ST change, $\mathrm{n}(\%)$ & 0 & 0 & 0 & $\begin{array}{l}1 \\
(10)\end{array}$ & $1(7)$ & 0 & 0 & $\begin{array}{l}2 \\
(13)\end{array}$ \\
\hline
\end{tabular}

\section{Figures}


A

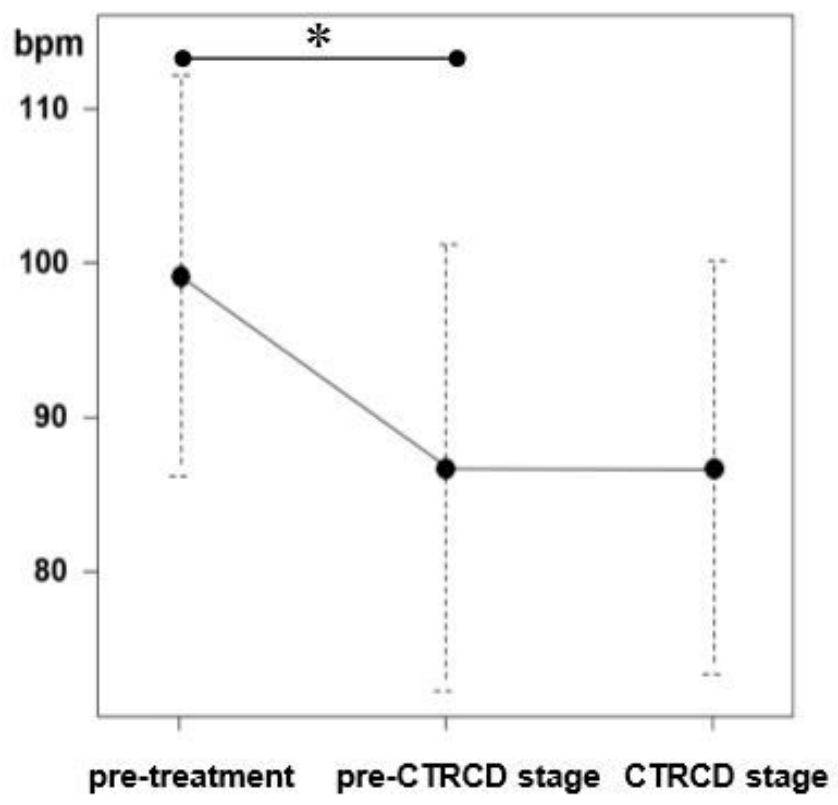

B

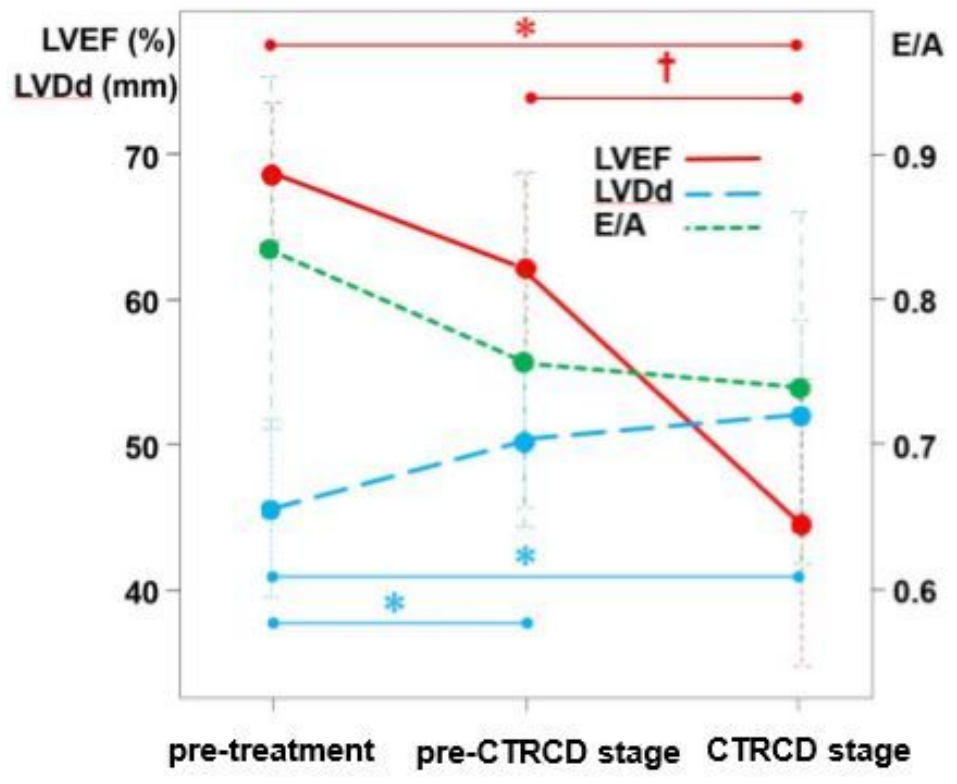

\section{Figure 1}

Fluctuations in the heart rate and echocardiographic indices in the pre-CTRCD and CTRCD stages. Heart rate significantly decreased in the pre-CTRCD and CTRCD stages compared with the pre-treatment levels (A). LVEF also significantly decreased, and LVDd increased in the pre-CTRCD and CTRCD stages compared with the pre-treatment values (B). CTRCD, cancer therapeutics-related cardiac dysfunction; LVEF, left ventricular ejection fraction; LVDd, left ventricular end-diastolic diameter; bpm, beats per minute; * $P<0.01 ;+P<0.05$ 


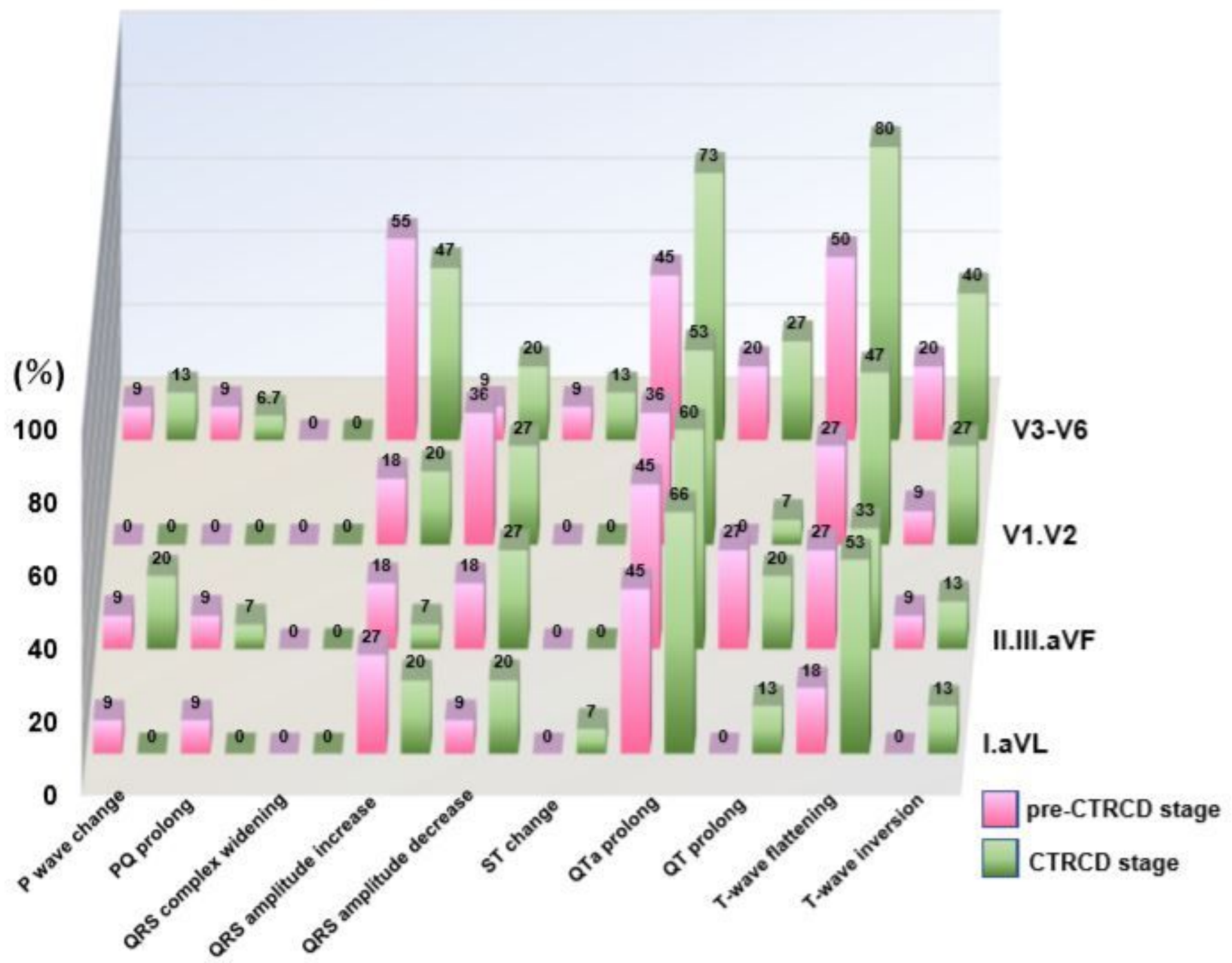

Figure 2

Electrocardiographic changes in the pre-CTRCD and CTRCD stages CTRCD, cancer therapeutics-related cardiac dysfunction 


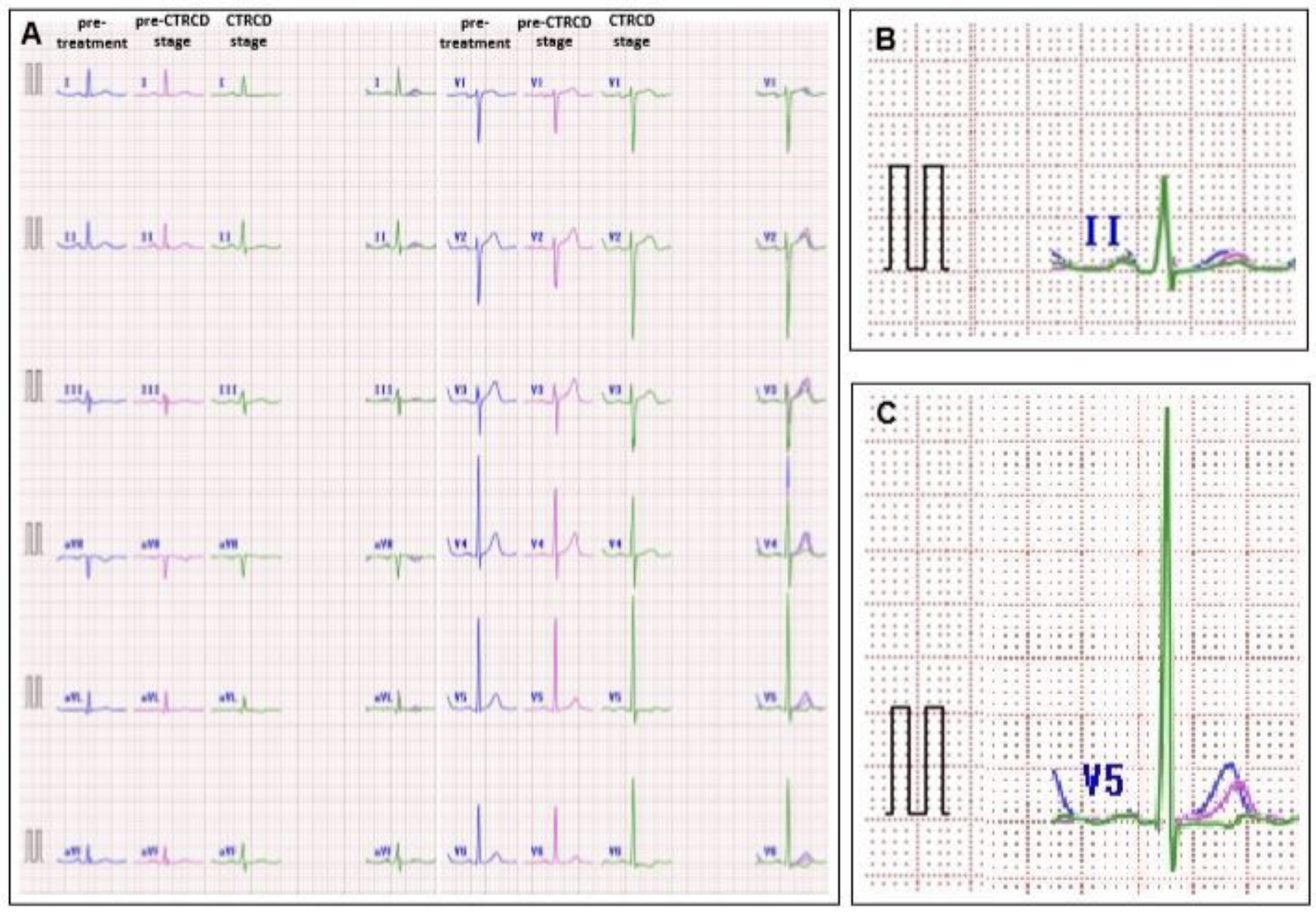

Figure 3

Representative ECG waveform fluctuations in patients with CTRCD Each waveform fluctuation in the pretreatment, pre-CTRCD, and CTRCD stages (A). QTa prolongation and T-wave flattening visually appeared in leads II (B) and V5 (C) in both pre-CTRCD and CTRCD stages. An increase in the QRS amplitude also appeared in $\mathrm{V} 5$ in the CTRCD stage. 